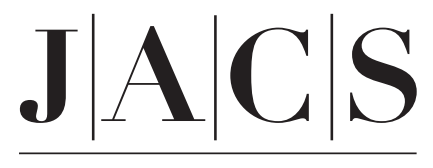

A R T I C L E S

Published on Web 07/10/2004

\title{
Agostic Interactions and Dissociation in the First Layer of Water on $\mathrm{Pt}(111)$
}

\author{
Timo Jacob and William A. Goddard III* \\ Contribution from the Materials and Process Simulation Center, Beckman Institute (139-74), \\ California Institute of Technology, Pasadena, California 91125 \\ Received January 6, 2004; E-mail:wag@wag.caltech.edu
}

\begin{abstract}
Recent quantum mechanical (QM) calculations for a monolayer of $\mathrm{H}_{2} \mathrm{O}$ on $\mathrm{Ru}(0001)$ suggested a novel stable structure with half the waters dissociated. However, different studies on $\mathrm{Pt}(111)$ suggested an undissociated bilayer structure in which the outer half of the water has the $\mathrm{OH}$ bonds toward the surface rather than the $\mathrm{O}$ lone pair. Since water layers on Pt are important in many catalytic processes (e.g., the fuel cell cathode), we calculated the energetics and structure of the first monolayer of water on the Pt(111) surface using QM [periodic slab using density functional calculations (DFT) with the PBE-flavor of exchangecorrelation functional]. We find that the fully saturated surface $(2 / 3 \mathrm{ML})$ has half the water almost parallel to the surface (forming a Pt-O Lewis acid-base bond), whereas the other half are perpendicular to the surface, but with the $\mathrm{H}$ down toward the surface (forming a $\mathrm{Pt}-\mathrm{HO}$ agostic bond). This leads to a net bond energy of $0.60 \mathrm{eV} /$ water $=13.8 \mathrm{kcal} / \mathrm{mol}$ (the standard ice model with the $\mathrm{H}$ up configuration of the water molecules perpendicular to the surface is less stable by $0.092 \mathrm{eV} /$ water $=2.1 \mathrm{kcal} / \mathrm{mol}$ ). We examined whether the partial dissociation of water proposed for $\mathrm{Ru}(0001)$ could occur on $\mathrm{Pt}(111)$. For the saturated water layer $(2 / 3 \mathrm{ML})$ we find a stable structure with half the $\mathrm{H}_{2} \mathrm{O}$ dissociated (forming $\mathrm{Pt}-\mathrm{OH}$ and $\mathrm{Pt}-\mathrm{H}$ covalent bonds), which is less favorable by only $0.066 \mathrm{eV} /$ water $=1.51 \mathrm{kcal} / \mathrm{mol}$. These results confirm the interpretation of combined experimental (XAS, XES, XPS) and theoretical (DFT cluster and periodic including spectrum calculations) studies, which find only the $\mathrm{H}$ down undissociated case. We find that the undissociated structure leads to a vertical displacement between the two layers of oxygens of $\sim 0.42 \AA$ (for both $\mathrm{H}$ down and $\mathrm{H}$ up). In contrast, the partially dissociated system leads to a flat structure with a separation of the oxygen layers of $0.08 \AA$. Among the partially dissociated systems, we find that all subsurface positions for the dissociated hydrogen are less favorable than adsorbing on top of the free Pt surface atom. Our results suggest that for less than $1 / 3 \mathrm{ML}$, clustering would be observed rather than ordered monolayer structures.
\end{abstract}

\section{Introduction}

Water plays a ubiquitous role in most important biological and chemical processes. Consequently, many investigations have been reported about the structure of water and water clusters in gas-phase and adsorbed on surfaces. Since water is present on nearly every real surface and plays an important role in electrochemical reactions on metals, the structures of the water overlayers on metals have been studied thoroughly. The most widely accepted model for the saturated first water layer on hexagonal metal surfaces has been the $H$-up bilayer structure (see Figure 1a) by Doering and Madey. ${ }^{1}$ This has half of the molecules (denoted as $\mathrm{H}_{2} \mathrm{O}$ ) binding directly to the surface using one lone pair orbital of the oxygen. The other half of the water molecules (denoted $\mathrm{HOH}$ ) are shifted away from the surface and stabilized by hydrogen bonds to the surface adsorbed water molecules (so that the non-hydrogen bonded $\mathrm{H}$ is up). This first ice $I_{h}$-like layer of water can stack additional layers to form a bulk ice- $\mathrm{I}_{\mathrm{h}}$ film.

In contrast to this bilayer picture of water on metals, low energy electron diffraction (LEED) experiments of water on

(1) Doering, D. L.; Madey, T. E. Surf. Sci. 1982, 123, 305.
$\mathrm{Ru}(0001)$ by Held and $\mathrm{Menzel}^{2}$ found no evidence for a large vertical displacement of half the water molecules. They measured only a small shift of $0.10 \pm 0.02 \AA$ (difference in the vertical position of the oxygens), whereas the bilayer structure would lead to ${ }^{2} 0.96 \AA$. A resolution of this problem for $\mathrm{Ru}(0001)$ was provided by Feibelman ${ }^{3}$, who concluded that neither the H-up bilayer structure nor the structure (hereafter, the $\mathrm{H}$-down bilayer structure) in which the up $\mathrm{H}$ of the $\mathrm{HOH}$ points down toward the surface would be stable (comparing calculated binding energies with the computed sublimation energy of ice). Instead, he used density functional calculations (DFT) and considered an alternative structure (half-dissociated layer), in which one $\mathrm{H}$ of each $\mathrm{HOH}$ water is dissociated, leading to a covalently bound $\mathrm{H}$ and hydroxyl (with the $\mathrm{H}$ bonded to another surface $\mathrm{Ru}$ ). This led to the small vertical oxygen displacement observed by experiments. Thus, Feibelman explained, the LEED-results in terms of partially dissociated water. It was suggested that this presence of water and hydroxyl molecules is supported by X-ray photoemission spectroscopy $(\mathrm{XPS})^{4}$ that find two different $\mathrm{O} 1 \mathrm{~s}$ peaks for water on

(2) Held, G.; Menzel, D. Surf. Sci. 1994, 316, 92.

(3) Feibelman, P. J. Science 2002, 295, 99. 

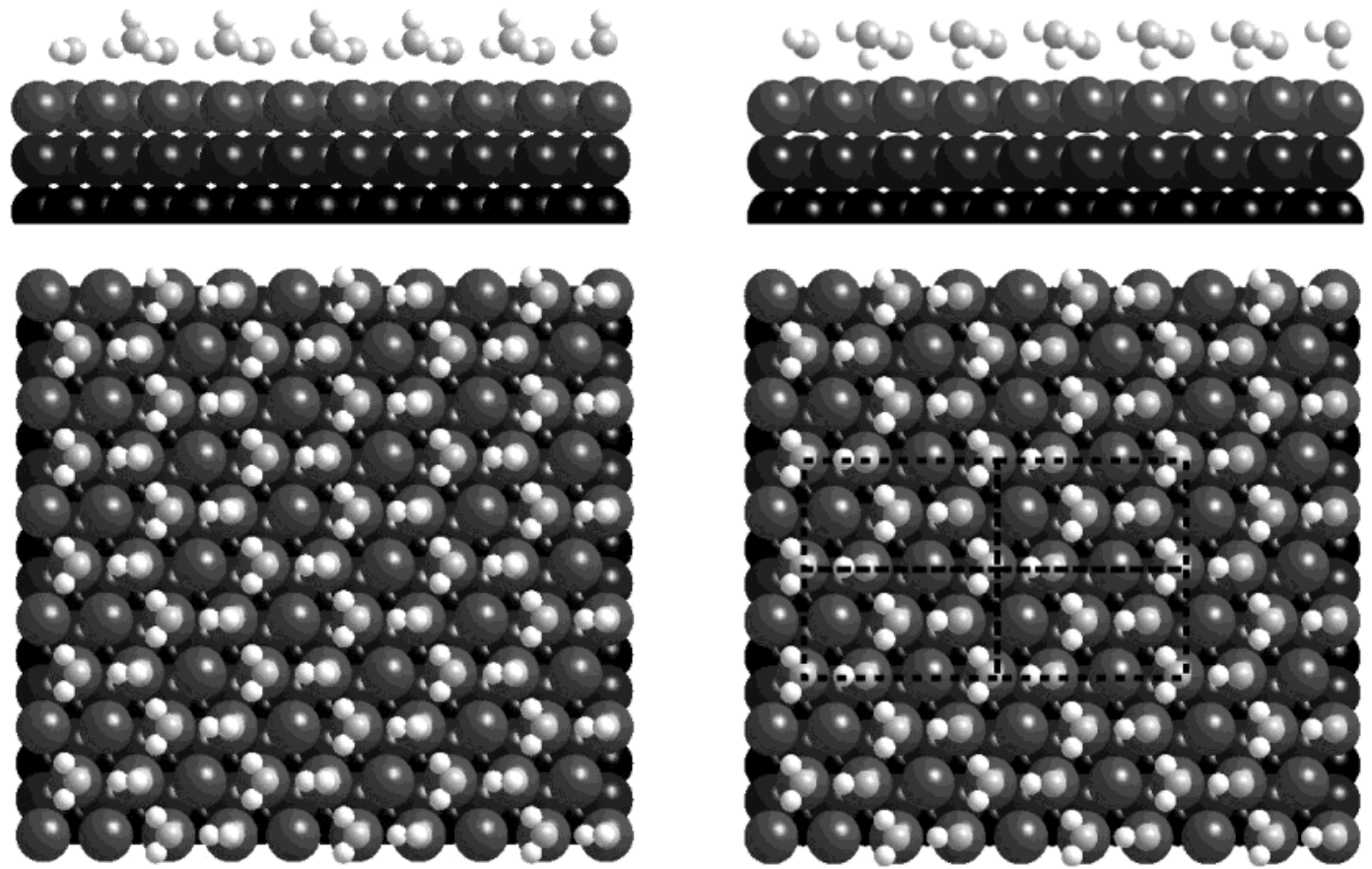

Figure 1. Ordered ice-like structures for $2 / 3 \mathrm{ML}$ of water on $\mathrm{Pt}(111)$. The left figure is the traditional bilayer structure (denoted as $\mathrm{H}$-up bilayer) with half of the water (denoted $\mathrm{H}_{2} \mathrm{O}$ ) parallel to the surface and bonded through the $\mathrm{O}$ lone pair directly to a $\mathrm{Pt}$ atom $(\mathrm{Pt}-\mathrm{O}=2.48 \AA$ ), whereas the other half of the water (denoted as $\mathrm{HOH}$ ) are perpendicular to the surface with the $\mathrm{O}$ displaced away from the surface by additional $0.42 \mathrm{~A}$ and the non- $\mathrm{H}-$-bonding hydrogens pointing up (away from the Pt). The right figure shows the $\mathrm{H}$-down bilayer structure in which the perpendicular waters (HOH) are in $\mathrm{H}$-down configuration forming an agostic $\mathrm{Pt}-\mathrm{HOH}$ bond $(\mathrm{Pt}-\mathrm{H}=2.11 \AA$ ). This leads to these $\mathrm{O}$ being displaced by $0.42 \AA$ from the first water layer. The dashed boxes in the right figure indicate the $\sqrt{3} \times 3$ surface unit cells. We find that the $\mathrm{H}$-up (left) configuration is $0.37 \mathrm{eV}$ per SUC less stable than the H-down, which has a total stability of $2.39 \mathrm{eV}$ with respect to 4 free water molecules.

$\mathrm{Ru}(0001)$. These studies raise the question of whether water on other close-packed surfaces might lead to similar partially dissociated or H-down water structures. However, O 1s photoemission spectra on $\mathrm{Ni}(111), \mathrm{Rh}(111), \mathrm{Cu}(111)$, or $\mathrm{Pt}(111)$ found no evidence for a partially dissociated structure. ${ }^{5,6}$

Since Pt catalysts are important for many catalytic reactions, including polymer electrolyte membrane fuel cells (PEM-FC), where water is formed at the cathode, we examined the structures and energies for the first layer of water on $\operatorname{Pt}(111)$ surface using periodic DFT methods. LEED, helium atom diffraction (HAD), and scanning tunneling microscopy (STM) agree that on $\mathrm{Pt}(111)$ water-saturation is achieved with $2 / 3$ of a monolayer (ML) to form a two-dimensional hexagonal ice-like structure. ${ }^{7-10} \mathrm{X}$-ray absorption/emission and XPS measurements by Ogasawara et al. ${ }^{6}$ confirm the coverage of $2 / 3 \mathrm{ML}$ and indicate that all water molecules are bound directly to the Pt surface without dissociation of hydrogen atoms. Furthermore, in conjunction of XAS experiments and periodic DFT calculations Ogasawara et al. characterized the $\mathrm{HOH}$ to have the H-down orientation (due to missing preedge peak in the spectra). From their periodic DFT calculations on the saturated overlayer, they confirmed the stable structure to be the H-down bilayer and found a Pt-O distance of $2.8 \AA$. However, an overall agreement between calculations and their XAS and XPS spectra was only achieved after forcing the $\mathrm{Pt}-\mathrm{O}$ distance (in the calculated system) to be $2.3-2.4 \AA$. Other periodic DFT calculations by Meng et al. ${ }^{11}$ computed the vibrational spectra of both, the H-up

(4) Pirug, G.; Ritke, C.; Bonzel, H. P. Surf. Sci. 1991, 241, 289

(5) Henderson, M. A. Surf. Sci. Rep. 2002, 46, 1.

(6) Ogasawara, O.; Brena, B.; Nordlund, D.; Nyberg, M.; Pelmenschikov, A.; Petterson, L. G. M.; Nilsson, A. Phys. Rev. Lett. 2002, 89(27), 276102.

(7) Glebov, A.; Graham, A. P.; Menzel, A.; Toennies, J. P. J. Chem. Phys. 1997, $106,9382$. and $\mathrm{H}$-down structure, to assist the experimental determination of the $\mathrm{HOH}$ orientation. However, they could not distinguish both structures from the vibrational spectra. They calculated a vertical oxygen displacement of $0.35 \AA$ for the H-down structure, compared to $0.63 \AA$ for the H-up bilayer structure.

\section{Method}

Our QM calculations used the SeqQuest ${ }^{12,13}$ periodic DFT program with Gaussian basis sets (rather than plane waves). We used the $\mathrm{PBE}^{14}$ Generalized Gradient Approximation (GGA) exchange-correlation functional, which has recently ${ }^{15}$ been shown to give reliable bond distances $(\Delta d=0.013 \AA)$ and energies $(\Delta E=0.09 \mathrm{kcal} / \mathrm{mol})$ for $\mathrm{H}$-bonded water dimers (compared to experiments).

The 62 core electrons of each Pt were replaced by a norm-conserving pseudopotential, ${ }^{16}$ leaving the $165 \mathrm{p}, 5 \mathrm{~d}$, and 6 s electrons to be treated explicitly. The different states were represented as follows:

- $6 \mathrm{~s}$ and 5d: each as a contracted basis function consisting of four Gaussians plus the most diffuse Gaussian of each set as additional function.

- 5p: a contracted basis function consisting of two Gaussians.

This contracted basis set of Gaussian functions was optimized for the Pt atom and different bulk structures (fcc, bcc, hcp, A15, Diamond, sc).

(8) Haq, S.; Harnett, J.; Hodgson, A. Surf. Sci. 2002, 505, 171.

(9) Morgenstern, M.; Michely, T.; Comsa, G. Phys. Rev. Lett. 1996, 77, 703

(10) Morgenstern, M.; Müller, J.; Michely, T.; Comsa, G. Z. Phys. Chem. 1997 $198,43$.

(11) Meng, S.; Xu, L. F.; Wang, E. G.; Gao, S. Phys. Rev. Lett. 2002, 89(17), 176104.

(12) ${ }^{1}$ Schultz, P. A., unpublished; A description of the method is in: Feibelman, P. J. Phys. Rev. B 1987, 35, 2626.

(13) Verdozzi, C.; Schultz, P. A.; Wu, R.; Edwards, A. H.; Kioussis, N. Phys. Rev. B 2002, 66, 125408

(14) Perdew, J. P.; Burke, K.; Ernzerhof, M. Phys. Rev. Lett. 1996, 88, 3865

(15) Xu, X.; Goddard, W. A., III. J. Phys. Chem. A 2004, 108(12), 2305.

(16) Hamann, D. R. Phys. Rev. B 1989, 40, 2980. 
All calculations used a three-layer slab to model the semi-infinite $\operatorname{Pt}(111)$ surface, with a $\sqrt{3} \times 3(4.806 \AA \times 8.324 \AA)$ surface unit cell (SUC) (6 Pt atoms per layer). The atoms of the bottom two layers were fixed at the experimental bulk crystal $\mathrm{Pt}-\mathrm{Pt}$ distance ${ }^{17}$ of 2.775 $\AA$, whereas the top Pt layer atoms and the water overlayer were fully optimized (to $<0.0025 \mathrm{eV} / \mathrm{SUC}$ ). To estimate the influence of the surface relaxation we also performed calculations allowing only the water molecules to relax, keeping all $\mathrm{Pt}$ atom positions fixed. There are hints that a saturated water layer forms an ordered ice-like structure on the $\operatorname{Pt}(111)$ surface, which would allow the SUC to be reduced to a $\sqrt{3} \times \sqrt{3}$ surface unit cell with two water molecules, however, we used an extended SUC $(\sqrt{3} \times 3)$ with four water molecules to reduce constraints on the system. This allowed the stability of the ordered structure to be studied as a function of coverage. All calculations used a converged Brillouin zone (BZ) sampling with $5 \times 3 k$-points.

\section{Results and Discussion}

Figures 2 and 3 show optimized structures for SUC having 1 to 4 waters $(1 / 6$ to $2 / 3 \mathrm{ML})$. All energetics and structural information are summarized in Tables $1-3$. It should be mentioned that distances for similar atoms (e.g., O of parallel waters) are combined. For example, $\mathrm{Pt}-\mathrm{O}_{1 / 3}=2.50,2.54 \AA$ combines $\mathrm{Pt}-\mathrm{O}_{1}=2.50 \AA$ and $\mathrm{Pt}-\mathrm{O}_{3}=2.54 \AA$.

With $1 / 6$ ML each water molecule (hereafter denoted as $\mathrm{H}_{2} \mathrm{O}$ ) binds on top of a Pt atom with its hydrogens oriented almost parallel to the surface (tilted $8.0^{\circ}$ ) above Pt bridge/3-fold sites. The $\mathrm{H}-\mathrm{O}-\mathrm{H}$ angle of $103.2^{\circ}$ is smaller than for free gas-phase water $\left(104.7^{\circ}\right)$. The $\mathrm{Pt}-\mathrm{O}$ distance is $2.46 \AA$, indicating a donor-acceptor bond from the $\mathrm{O}$ lone pair to a $\mathrm{Pt}$ atom. The net bond energy is $0.38 \mathrm{eV} /$ water $=8.69 \mathrm{kcal} / \mathrm{mol}$.

In addition, we considered the case in which the surface water dissociates to form surface $\mathrm{OH}$ and surface $\mathrm{H}$. The best case is to have both $\mathrm{OH}$ and $\mathrm{H}$ at on top sites. The total energy of this system is $0.43 \mathrm{eV}$ higher than the undissociated case. The calculated energy to dissociate $\mathrm{H}_{2} \mathrm{O}$ into $\mathrm{OH}$ and $\mathrm{H}$ is $5.24 \mathrm{eV}$ (experimental values $D_{0}=5.099 \pm 0.003 \mathrm{eV}^{18}$ and $D_{\mathrm{e}}=5.45$ $\mathrm{eV}$ ) so that the sum of the $\mathrm{Pt}-\mathrm{H}$ and $\mathrm{Pt}-\mathrm{OH}$ energies is 4.81 $\mathrm{eV}$. This can be compared to separate calculations on the slab of $\mathrm{D}(\mathrm{H} / \mathrm{Pt})=2.65 \mathrm{eV}$ and $\mathrm{D}(\mathrm{OH} / \mathrm{Pt})=2.12 \mathrm{eV}$. Thus, adding the separate surface bonds to predict the coadsorbed cases is only $0.04 \mathrm{eV}$ too weak. For isolated $\mathrm{Pt}_{35}$ clusters we find a $\mathrm{Pt}-$ $\mathrm{OH}$ bond energy of $2.06 \mathrm{eV}(\mathrm{Pt}-\mathrm{O}$ bond $=1.979 \AA)$ and a $\mathrm{Pt}-\mathrm{H}$ bond energy of $2.73 \mathrm{eV}(\mathrm{Pt}-\mathrm{H}$ bond $=1.544 \AA$ ).

Adding a second water to the SUC $(1 / 3 \mathrm{ML})$, the stable structure in Figure $2 \mathrm{~b}$ has essentially the same distances and angles as for $1 / 6 \mathrm{ML}$, with all waters bound on top of a Pt atom and are oriented almost parallel to the surface $\left(S^{\|}=8.0-8.1^{\circ}\right)$. This ordered structure has all $\mathrm{O}-\mathrm{O}$ distances at $\sim 4.7 \AA$, too large for $\mathrm{H}$ bonding. This leads to adsorption energy of 0.76 $\mathrm{eV}$ (per SUC), exactly twice the energy for $1 / 6 \mathrm{ML}$.

Since the above structure has shortest $\mathrm{O}-\mathrm{O}$ distances of $\sim 4.7$ $\AA$, which is too large for $\mathrm{H}$ bonding, we also considered adding the second water $\left(\mathrm{O}_{2}\right)$ with its $\mathrm{H}-\mathrm{O}-\mathrm{H}$ plane perpendicular to the surface, so as to form hydrogen bonds to two first layer waters. This leads to two possible orientations of the $\mathrm{H}$ atoms: $\mathrm{H}$-up and H-down (toward the Pt) (optimized structures are shown in Figure 2). The H-up configuration conforms with the

(17) Kittel, Ch. Einführung in die Festkörperphysik; R. Oldenbourg Verlag: München, 1991

(18) Ruscic, B.; Wagner, A. F.; Harding, L. B.; Asher, R. L.; Feller, D.; Dixon, D. A.; Peterson, K. A.; Song, Y.; Qian, X. M.; Ng, C. Y.; Liu, J. B.; Chen, W. W. J. Phys. Chem. A 2002, $106(11), 2727$. traditional bilayer model for $2 / 3$ ML (Figure 1a) and is $0.19 \mathrm{eV}$ per SUC lower in energy than the single layer structure, but we find that the $\mathrm{H}$ down structure (as in Figure $1 \mathrm{~b}$ ) is $0.17 \mathrm{eV}$ more stable than $\mathrm{H}$ up, due to the additional agostic interaction ${ }^{19-21}$ of the down $\mathrm{OH}$ with a surface Pt. The vertical distance between $\mathrm{O}_{1}$ - and $\mathrm{O}_{2}$-water is $0.42 \AA$ for $\mathrm{H}$ up and $0.68 \AA$ for $\mathrm{H}$ down. This H-down bilayer structure is different from the structure (partially dissociated) proposed by Feibelman for water on $\mathrm{Ru}$ (0001), but consistent with the structure proposed by Ogasawara et al. We find that the $\mathrm{O}_{1}$ parallel oriented $\mathrm{H}_{2} \mathrm{O}$ (notation see Figures) forms zigzag water chains so that each $\mathrm{H}_{2} \mathrm{O}$ donates hydrogen bonds to two $\mathrm{HOH}$, leading to an opening of the bond angle to $105.2^{\circ}$.

In the $\mathrm{H}$-up structure the $\mathrm{HOH}$ has a $\mathrm{Pt}-\mathrm{O}_{1}$ distance of 2.41 $\AA$, just as for $1 / 6 \mathrm{ML}$, whereas the $\mathrm{Pt}-\mathrm{O}_{2}$ distance is $2.96 \AA$. However, for $\mathrm{H}$-down $\mathrm{Pt}-\mathrm{O}_{2}$ increases to $3.04 \AA$ to accommodate the agostic $\mathrm{H}$, which ends up just $2.03 \AA$ from the Pt, whereas $\mathrm{Pt}-\mathrm{O}_{1}$ decreases to just $2.22 \AA$. This can be compared to the covalent bond of $\mathrm{OH}$ to the surface, where $\mathrm{Pt}-\mathrm{OH}=$ $1.99 \AA$.

The $\mathrm{O}_{1}$-water prefers to orient its hydrogens toward the $\mathrm{O}$ atoms of the neighboring $\mathrm{O}_{2}$-waters, causing the tilting of the $\mathrm{O}_{1}$-water. For the $\mathrm{H}$-up structure the $\mathrm{O}_{1}$-water tilts by $10.3^{\circ}$, whereas the $\mathrm{H}$-down structure leads to a $24.5^{\circ}$ tilt of the $\mathrm{H}-\mathrm{O}-\mathrm{H}$ plane due to the $0.19 \AA$ shorter $\mathrm{Pt}-\mathrm{O}_{1}$ length in the $\mathrm{H}$ down system. This leads to a hydrogen bond with $\mathrm{O}-\mathrm{O}$ distances of $2.89 \AA$ (H-down) and $2.87 \AA$ (H-up) both shorter than for the free dimer $(3.00 \AA)$ and longer than for hexagonal ice $(2.74 \AA){ }^{22}$

We find that bonding an isolated parallel oriented $\mathrm{H}_{2} \mathrm{O}$ to the surface through the lone pair is $\mathrm{Pt}-\mathrm{H}_{2} \mathrm{O}=0.38 \mathrm{eV}$ and that binding two isolated $\mathrm{H}_{2} \mathrm{O}$ parallel to the surface the total bond energy is $0.76 \mathrm{eV}$. This bond additivity suggests that we decompose the surface binding energies into simple additive quantities. Thus, for the H-up bilayer with a total binding energy of $0.93 \mathrm{eV}$ and 2 hydrogen bonds (HB) per cell, we write $0.93=0.38+2 \mathrm{HB}$, leading to $\mathrm{HB}=0.28 \mathrm{eV}=6.5 \mathrm{kcal} /$ mol, a little above the bond energy of water dimer, $5.02 \mathrm{kcal} /$ mol. Then considering the more stable $\mathrm{H}$-down bilayer, we get $1.12=0.38+2 \mathrm{HB}+\mathrm{Pt}-\mathrm{HOH}$, indicating that the surface agostic bond is $\mathrm{Pt}-\mathrm{HOH}=0.18 \mathrm{eV}=4.2 \mathrm{kcal} / \mathrm{mol}$, which is weaker to the normal isolated $\mathrm{H}_{2} \mathrm{O}$ bond to $\mathrm{Pt}$ of $0.38 \mathrm{eV}$.

To illustrate the use of such bond additivity quantities, consider the likely structure for an isolated cluster of 4 waters on the surface. This would have 3 parallel waters bonded to $\mathrm{Pt}$ atoms with one $\mathrm{HOH}$ down water making a $\mathrm{HB}$ to all three neighboring molecules. This leads to a total bond energy of

$$
3 \times 0.38+0.18+3 \mathrm{HB}=2.16 \text { or } 0.54 \mathrm{eV} / \mathrm{H}_{2} \mathrm{O}
$$

Similar considerations lead to a cluster of 13 waters with a total energy of

$$
6 \times 0.38+7 \times 0.18+15 \mathrm{HB}=7.74 \text { or } 0.60 \mathrm{eV} / \mathrm{H}_{2} \mathrm{O}
$$

and a cluster of 24 waters with a total energy of

$$
12 \times 0.38+12 \times 0.18+30 \mathrm{HB}=15.12 \text { or } 0.63 \mathrm{eV} / \mathrm{H}_{2} \mathrm{O}
$$

(19) Brookhart, M.; Green, M. L. H. J. Organomet. Chem. 1983, 250, 395. 
(a)

$\mathrm{Pt} / \mathrm{H}_{2} \mathrm{O}$

$6 \mathrm{O}_{1}$
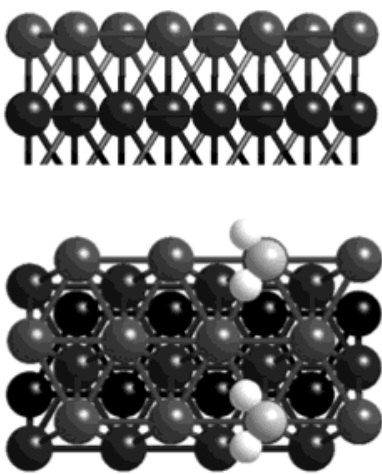

(d)

$\mathrm{Pt} / \mathrm{H}_{2} \mathrm{O}-\mathrm{HOH}$ (H down)
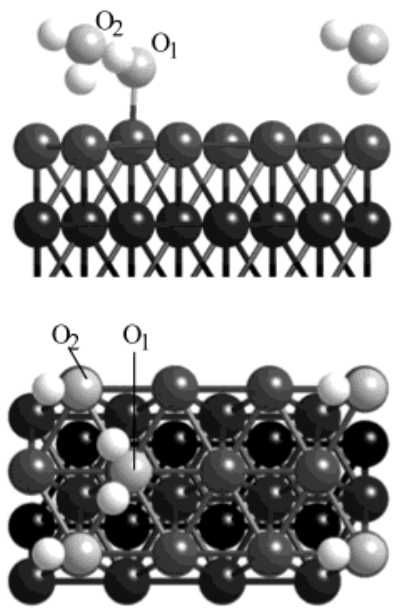

(g)
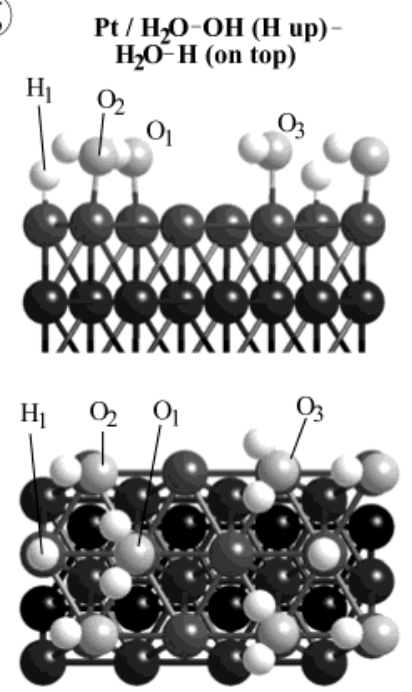

(b)
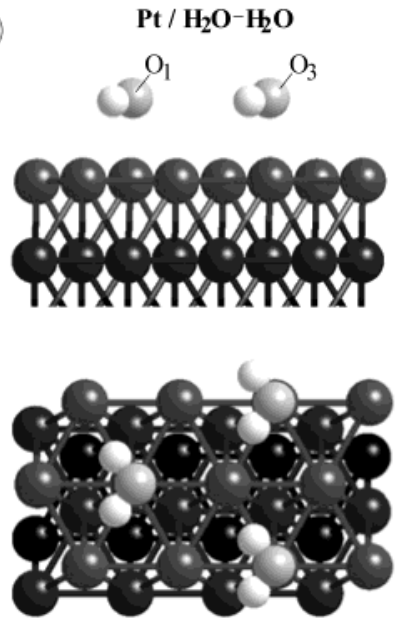

(e)

$\mathrm{Pt} / \mathrm{H}_{2} \mathrm{O}-\mathrm{HOH}$ (H up) $-\mathrm{H}_{2} \mathrm{O}$
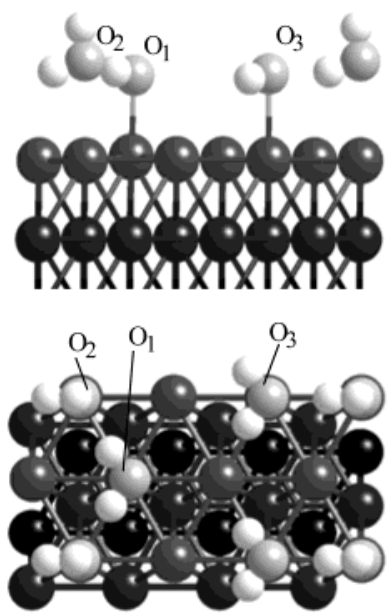

(h)

Pt / OH (H up)-HOH (H up) $\mathrm{H}_{2} \mathrm{O}-\mathrm{H}$ (on top)
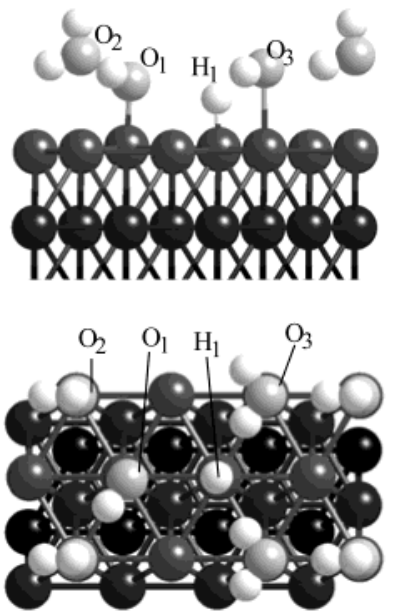

(c)
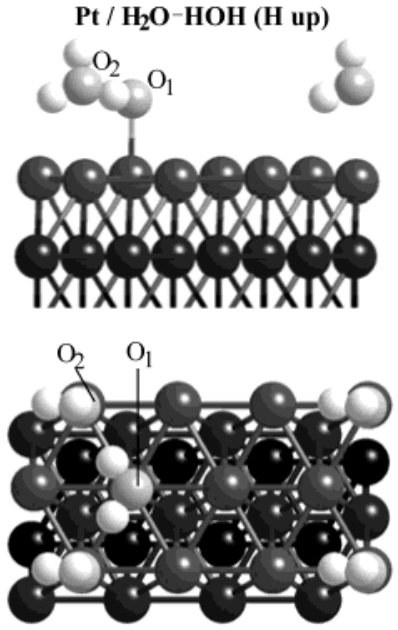

(f)

$\mathrm{Pt} / \mathrm{H}_{2} \mathrm{O}-\mathrm{HOH}(\mathrm{H}$ down $)-\mathrm{H}_{2} \mathrm{O}$
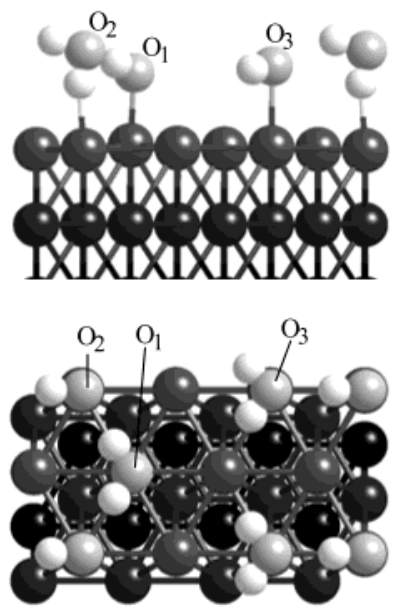

(i) $\mathrm{Pt} / \mathrm{OH}$ (H up)-HOH (H down)$\mathrm{H}_{2} \mathrm{O}-\mathrm{H}$ (on top)
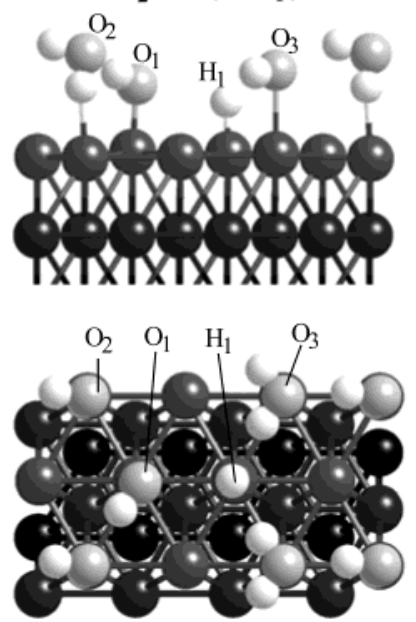

Figure 2. Side and top views of optimized structures with $1 / 6$ ML (a), $1 / 3$ ML $(b-d)$, and $1 / 2$ ML (e-i). For clarity, we show slightly more than the SUC. Structure $\mathrm{d}$ is the most stable for $1 / 3 \mathrm{ML}$ and structure $\mathrm{f}$ is the most stable for $1 / 2 \mathrm{ML}$. Structures $\mathrm{g}, \mathrm{h}$, and i have a $\mathrm{HOH}$ or $\mathrm{H}_{2} \mathrm{O}$ partially dissociated.

This can be compared to the experimental value at low coverage of $0.52 \mathrm{eV} .{ }^{23}$ Since these cluster energies lead to energies per

(20) Brookhart, M.; Green, M. L. H.; Wong, L. L. Prog. Inorg. Chem. 1988, 36,1 . water more stable than that of our ordered chain structure for $1 / 3 \mathrm{ML}$, we expect that at this coverage, the surface waters may form small islands rather than an ordered overlayer. This agrees

(21) McGrady, G. S.; Downs, A. J. Coord. Chem. Rev. 2000, 197, 95. 
(a)

$\mathrm{Pt} / \mathrm{H}_{2} \mathrm{O}-\mathrm{HOH}$ (H up)-

$\mathrm{H}_{2} \mathrm{O}-\mathrm{HOH}$ (H up)
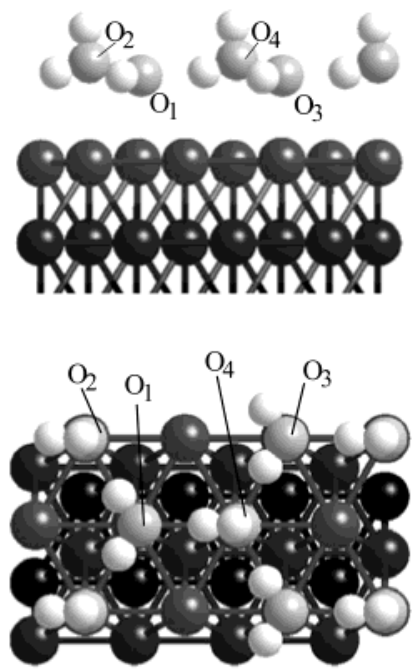

(b)

$\mathrm{Pt} / \mathrm{H}_{2} \mathrm{O}-\mathrm{HOH}$ (H down) $\mathrm{H}_{2} \mathrm{O}-\mathrm{HOH}$ (H down)
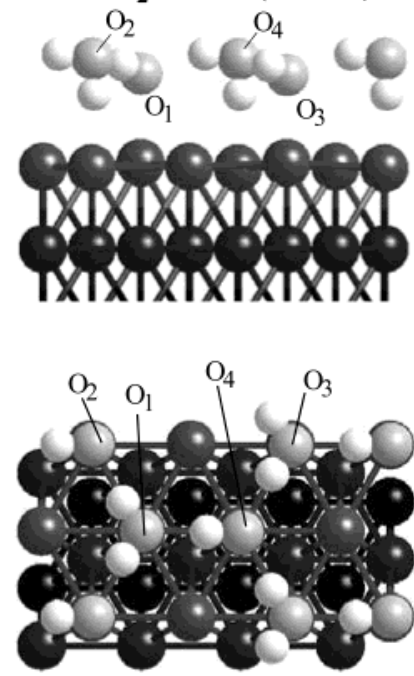

(C)

$\mathrm{Pt} / \mathrm{H}_{2} \mathrm{O}-\mathrm{OH}$ (H up)- $\mathrm{H}$ (on top)-

$\mathrm{H}_{2} \mathrm{O}-\mathrm{OH}$ (H up)-H (on top)
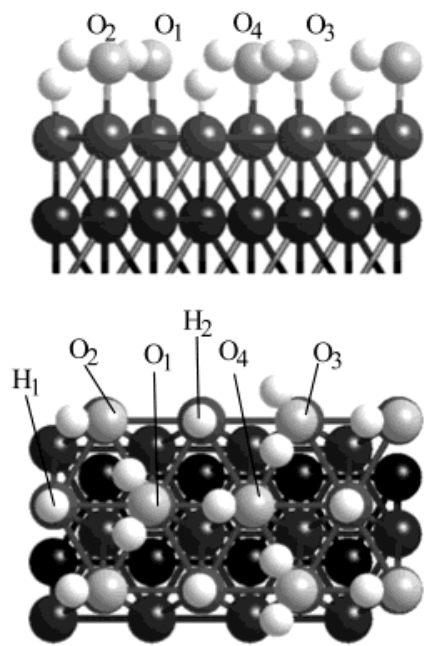

(d)

$\mathrm{Pt} / \mathrm{H}_{2} \mathrm{O}-\mathrm{OH}$ (H up)- $\mathrm{H}$ (sub 1)-

$\mathrm{H}_{2} \mathrm{O}^{-} \mathrm{OH}$ (H up)- $\mathrm{H}$ (sub 1)

$\mathrm{O}_{2} \mathrm{O}_{1} \mathrm{O}_{4} \mathrm{O}_{3}$
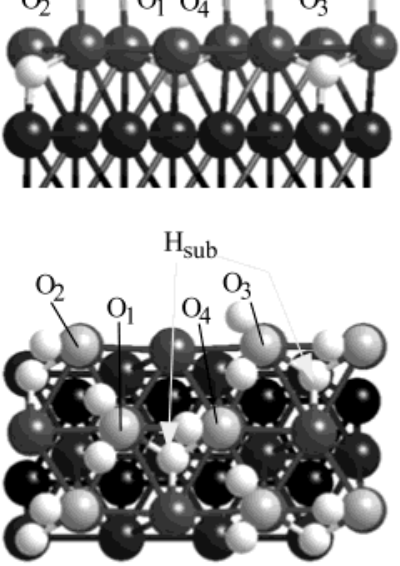

(e)

$\mathrm{Pt} / \mathrm{H}_{2} \mathrm{O}-\mathrm{OH}$ (H up)- $\mathrm{H}$ (sub 2)-

$\mathrm{H}_{2} \mathrm{O}-\mathrm{OH}$ (H up)- $\mathrm{H}$ (sub 2)
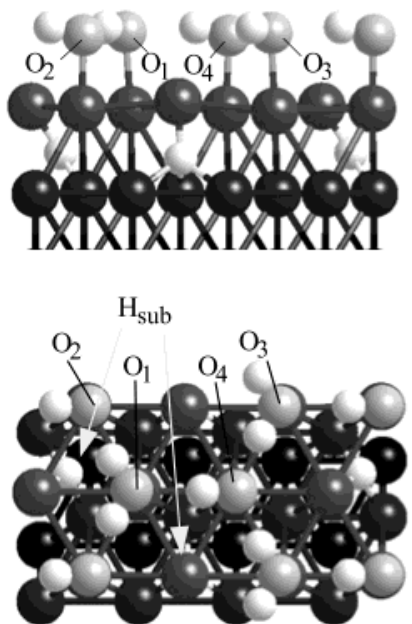

(f)

$\mathrm{Pt} / \mathrm{OH}$ (H up) $-\mathrm{H}_{2} \mathrm{O}-\mathrm{H}$ (sub 3)$\mathrm{H}_{2} \mathrm{O}-\mathrm{OH}$ (H up)-H (sub 3)

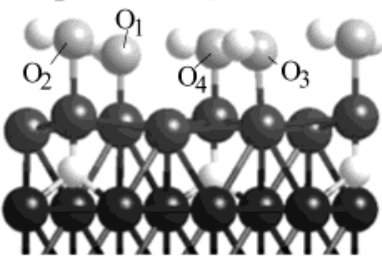

$\mathrm{H}_{\text {sub }}$

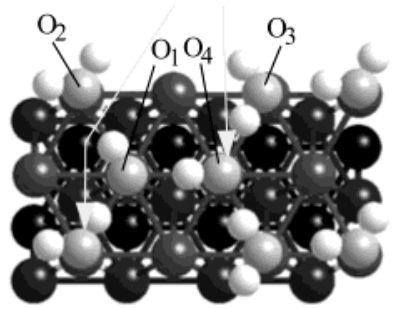

g)

$\mathrm{Pt} / \mathrm{H}_{2} \mathrm{O}-\mathrm{OH}$ (H up)- $\mathrm{H}$ (sub 3)-

$\mathrm{H}_{2} \mathrm{O}-\mathrm{OH}$ (H up)- $\mathrm{H}$ (sub 3)

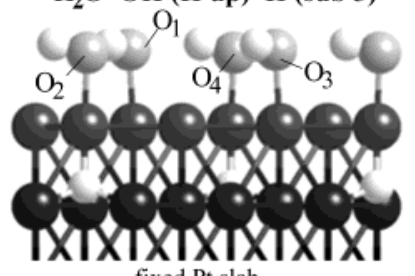

fixed Pt slab

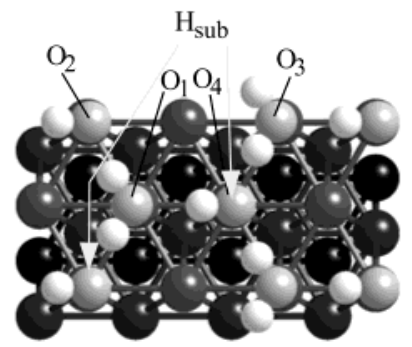

Figure 3. Side and top views of optimized structures with $2 / 3$ ML. For clarity, we show slightly more than the SUC. In structures $d-g$ the dissociated $H$ atoms are subsurface (beneath the first Pt surface layer, see arrows). Structure b is the optimum structure for $2 / 3 \mathrm{ML}$ and $\mathrm{c}$ is the optimum structure with dissociated water.

Table 1. Energetics and Structures for the Reference Systems To Calculate Binding Energies

\begin{tabular}{|c|c|c|c|c|}
\hline system & $1^{\text {st }}$ Pt-layer & $E_{\text {tot }}[\mathrm{h}]$ & distances $[\AA]$ & angles [deg] \\
\hline $\mathrm{H}_{2} \mathrm{O}$ molecule & relaxed & -17.1682 & $\mathrm{O}-\mathrm{H}=0.960$ & $\mathrm{H}-\mathrm{O}-\mathrm{H}=104.7$ \\
\hline $\mathrm{OH}$ molecule & relaxed & -16.4860 & $\mathrm{O}-\mathrm{H}=0.981$ & \\
\hline $\mathrm{H}$ atom & & -0.5129 & & \\
\hline \multirow[t]{2}{*}{ Pt-slab } & fixed & -473.5829 & $\begin{array}{l}\mathrm{Pt}-\mathrm{Pt}=2.775 \\
\text { interlayer }=2.261\end{array}$ & \\
\hline & relaxed & -473.5881 & $\begin{array}{l}\mathrm{Pt}-\mathrm{Pt}=2.775 \\
\text { interlayer }=2.32-2.36\end{array}$ & \\
\hline
\end{tabular}

with the scanning tunneling microscopy (STM) studies by Mitsui et al. who studied water diffusion on hexagonal $\operatorname{Pd}(111)$ and found clustering rather than ordered structures at low coverage. ${ }^{24}$

(22) Materer, N.; Starke, U.; Barbieri, A.; Van Hove, M. A.; Kroes, G.-J.; Minot, C.; Somorjai, G. A. J. Phys. Chem. 1995, 99, 6267.

(23) Thiel, P. A.; Madey, T. E. Surf. Sci. Rep. 1987, 7, 211.
For $1 / 2$ ML, we find a structure with the H-down chain as for $1 / 3 \mathrm{ML}$, but in addition, there is a second parallel $\mathrm{H}_{2} \mathrm{O}$ (labeled $\mathrm{O}_{3}$-water), which is an appendage on this chain and accepts a hydrogen bond from one $\mathrm{HOH}$.

(24) Mitsui, T.; Rose, M. K.; Fomin, E.; Ogletree, D. F.; Slameron, M. Science 2002, $297,1850$. 
Table 2. Binding Energies and Structures for Water on Pt(111) Ranging from $1 / 6$ to $1 / 2 \mathrm{ML}\left(1 \text { to } 3 \mathrm{H}_{2} \mathrm{O} \text { Per SUC }\right)^{a}$

\begin{tabular}{|c|c|c|c|}
\hline system (always on Pt) & $E_{\text {bind }}[\mathrm{eV}]$ & distances $[\AA]$ & angles [deg] \\
\hline $\mathrm{H}_{2} \mathrm{O}$ & 0.377 & $\mathrm{Pt}-\mathrm{O}_{1}=2.46$ & $\mathrm{H}-\mathrm{O}_{1}-\mathrm{H}=103.2$ \\
\hline $1 / 6 \mathrm{ML}$ & & $\mathrm{O}_{1}-\mathrm{H}=0.98$ & $\mathrm{~S}^{\perp}-\left(\mathrm{Pt}-\mathrm{O}_{1}\right)=0.8$ \\
\hline Figure $2 \mathrm{a}$ & & & $\mathrm{S}^{\mathrm{II}}-\left(\mathrm{H}-\mathrm{O}_{1}-\mathrm{H}\right)=8.1$ \\
\hline $\mathrm{H}_{2} \mathrm{O}-\mathrm{H}_{2} \mathrm{O}$ & 0.755 & $\mathrm{Pt}-\mathrm{O}_{1 / 3}=2.46,2.47$ & $\mathrm{H}-\mathrm{O}_{1 / 3}-\mathrm{H}=103.0,106.1$ \\
\hline $1 / 3 \mathrm{ML}$ & & $\mathrm{O}_{1 / 3}-\mathrm{H}=0.98$ & $\mathrm{~S}^{\perp}-\left(\mathrm{Pt}-\mathrm{O}_{1 / 3}\right)=0.5,1.4$ \\
\hline Figure $2 b$ & & $\mathrm{O}_{1}-\mathrm{O}_{3}=4.72$ & $\mathrm{~S}^{\| I}-\left(\mathrm{H}-\mathrm{O}_{1 / 3}-\mathrm{H}\right)=8.0$ \\
\hline $\mathrm{H}_{2} \mathrm{O}-\mathrm{HOH}(\mathrm{H}$ up $)$ & 0.943 & $\mathrm{Pt}-\mathrm{O}_{1}=2.41$ & $\mathrm{H}-\mathrm{O}_{1}-\mathrm{H}=107.8$ \\
\hline $1 / 3 \mathrm{ML}$ & & $\mathrm{Pt}-\mathrm{O}_{2}=2.96$ & $\mathrm{H}-\mathrm{O}_{2}-\mathrm{H}=103.4$ \\
\hline \multirow[t]{4}{*}{ Figure $2 \mathrm{c}$} & & $\mathrm{O}_{1}-\mathrm{H}=0.99$ & $\mathrm{~S}^{\perp}-\left(\mathrm{O}_{2}-\mathrm{H}_{\mathrm{up}}\right)=10.2$ \\
\hline & & $\mathrm{O}_{2}-\mathrm{H}_{\text {up } / \text { down }}=0.98,0.99$ & $\mathrm{~S}^{\perp}-\left(\mathrm{Pt}-\mathrm{O}_{2}\right)=1.1$ \\
\hline & & $\mathrm{O}_{1}-\mathrm{O}_{2}=2.87$ & $\mathrm{~S}^{\perp}-\left(\mathrm{Pt}-\mathrm{O}_{1}\right)=0.8$ \\
\hline & & & $\mathrm{S}^{\mathrm{II}}-\left(\mathrm{H}-\mathrm{O}_{1}-\mathrm{H}\right)=10.4$ \\
\hline \multirow{6}{*}{$\begin{array}{l}\mathrm{H}_{2} \mathrm{O}-\mathrm{HOH} \text { (H down) } \\
1 / 3 \mathrm{ML} \\
\text { Figure } 2 \mathrm{~d} \\
\text { (most stable structure } \\
\text { for } 1 / 3 \mathrm{ML} \text { ) }\end{array}$} & 1.118 & $\mathrm{Pt}-\mathrm{O}_{1}=2.22$ & $\mathrm{H}-\mathrm{O}_{1}-\mathrm{H}=105.2$ \\
\hline & & $\mathrm{Pt}-\mathrm{O}_{2}=3.04$ & $\mathrm{H}-\mathrm{O}_{2}-\mathrm{H}=99.6$ \\
\hline & & $\mathrm{O}_{1}-\mathrm{H}=1.00$ & $\mathrm{~S}^{\| \prime}-\left(\mathrm{O}_{2}-\mathrm{H}_{\text {up }}\right)=18.2$ \\
\hline & & $\mathrm{O}_{2}-\mathrm{H}_{\text {up/down }}=0.98,1.02$ & $\mathrm{~S}^{\perp-}\left(\mathrm{Pt}-\mathrm{O}_{2}\right)=0.8$ \\
\hline & & $\mathrm{O}_{1}-\mathrm{O}_{2}=2.90$ & $\mathrm{~S}^{\perp-}\left(\mathrm{Pt}-\mathrm{O}_{1}\right)=2.8$ \\
\hline & & & $\mathrm{S}^{\mathrm{Il}}-\left(\mathrm{H}-\mathrm{O}_{1}-\mathrm{H}\right)=24.5$ \\
\hline \multirow{7}{*}{$\begin{array}{l}\mathrm{H}_{2} \mathrm{O}-\mathrm{HOH}(\mathrm{H} \text { up })-\mathrm{H}_{2} \mathrm{O} \\
\quad 1 / 2 \mathrm{ML} \\
\quad \text { Figure } 2 \mathrm{e}\end{array}$} & 1.434 & $\mathrm{Pt}-\mathrm{O}_{1}=2.41$ & $\mathrm{H}-\mathrm{O}_{1}-\mathrm{H}=107.8$ \\
\hline & & $\mathrm{Pt}-\mathrm{O}_{2}=2.95$ & $\mathrm{H}-\mathrm{O}_{2}-\mathrm{H}=101.4$ \\
\hline & & $\mathrm{Pt}-\mathrm{O}_{3}=2.55$ & $\mathrm{H}-\mathrm{O}_{3}-\mathrm{H}=103.9$ \\
\hline & & $\mathrm{O}_{1 / 3}-\mathrm{H}=0.98-0.99$ & $\mathrm{~S}^{\perp}-\left(\mathrm{O}_{2}-\mathrm{H}_{\mathrm{up}}\right)=8.3$ \\
\hline & & $\mathrm{O}_{2}-\mathrm{H}_{\mathrm{up}}=0.98$ & $\mathrm{~S}^{\perp-}\left(\mathrm{Pt}-\mathrm{O}_{2}\right)=1.5$ \\
\hline & & $\mathrm{O}_{2}-\mathrm{H}_{\text {down }}=1.00$ & $\mathrm{~S}^{\perp-}\left(\mathrm{Pt}-\mathrm{O}_{1}\right)=0.6$ \\
\hline & & $\mathrm{O}_{1}-\mathrm{O}_{2}=2.87$ & $\mathrm{~S}^{\prime \prime}-\left(\mathrm{H}-\mathrm{O}_{1 / 3}-\mathrm{H}\right)=12.5,13.3$ \\
\hline \multirow{8}{*}{$\begin{array}{l}\mathrm{H}_{2} \mathrm{O}-\mathrm{HOH}(\mathrm{H} \text { down })-\mathrm{H}_{2} \mathrm{O} \\
1 / 2 \mathrm{ML} \\
\text { Figure } 2 \mathrm{f} \\
\text { (most stable structure } \\
\quad \text { for } 1 / 2 \mathrm{ML} \text { ) }\end{array}$} & 1.571 & $\mathrm{Pt}-\mathrm{O}_{1}=2.22$ & $\mathrm{H}-\mathrm{O}_{1}-\mathrm{H}=105.2$ \\
\hline & & $\mathrm{Pt}-\mathrm{O}_{2}=3.05$ & $\mathrm{H}-\mathrm{O}_{2}-\mathrm{H}=99.8$ \\
\hline & & $\mathrm{Pt}-\mathrm{O}_{3}=2.49$ & $\mathrm{H}-\mathrm{O}_{3}-\mathrm{H}=103.5$ \\
\hline & & $\mathrm{O}_{1 / 3}-\mathrm{H}=0.98-1.00$ & $\mathrm{~S}^{\| \prime}-\left(\mathrm{O}_{2}-\mathrm{H}_{\mathrm{up}}\right)=15.8$ \\
\hline & & $\mathrm{O}_{2}-\mathrm{H}_{\text {up/down }}=0.98,1.01$ & $\mathrm{~S}^{\perp}-\left(\mathrm{Pt}-\mathrm{O}_{2}\right)=0.3$ \\
\hline & & $\mathrm{O}_{1}-\mathrm{O}_{2}=2.89$ & $\mathrm{~S}^{\perp-}\left(\mathrm{Pt}-\mathrm{O}_{1}\right)=2.1$ \\
\hline & & & $\mathrm{S}^{\mathrm{II}}-\left(\mathrm{H}-\mathrm{O}_{1}-\mathrm{H}\right)=28.4$ \\
\hline & & & $\mathrm{S}^{\mathrm{Il}}-\left(\mathrm{H}-\mathrm{O}_{3}-\mathrm{H}\right)=9.9$ \\
\hline \multirow{7}{*}{$\begin{array}{l}\mathrm{H}_{2} \mathrm{O}-\mathrm{OH}(\mathrm{H} \text { up })-\mathrm{H}_{2} \mathrm{O}-\mathrm{H} \text { (top) } \\
1 / 2 \mathrm{ML} \\
\text { Figure } 2 \mathrm{~g} \\
\text { (most stable dissociated } \\
\quad \text { structure for } 1 / 2 \mathrm{ML} \text { ) }\end{array}$} & 1.375 & $\mathrm{Pt}-\mathrm{O}_{1}=2.12$ & $\mathrm{H}-\mathrm{O}_{1}-\mathrm{H}=112.4$ \\
\hline & & $\mathrm{Pt}-\mathrm{O}_{2}=2.11$ & $\mathrm{H}-\mathrm{O}_{3}-\mathrm{H}=107.5$ \\
\hline & & $\mathrm{Pt}-\mathrm{O}_{3}=2.30$ & $\mathrm{Pt}-\mathrm{O}_{2}-\mathrm{H}_{\text {up }}=99.9$ \\
\hline & & $\mathrm{Pt}-\mathrm{H}_{1}=1.55$ & $\mathrm{~S}^{\perp}-\left(\mathrm{Pt}-\mathrm{O}_{2}\right)=5.5$ \\
\hline & & $\mathrm{O}_{2 / 3}-\mathrm{H}=0.98$ & $\mathrm{~S}^{\perp}-\left(\mathrm{Pt}-\mathrm{O}_{1}\right)=3.2$ \\
\hline & & $\mathrm{O}_{1}-\mathrm{H}=1.02$ & $\mathrm{~S}^{\mathrm{Il}}-\left(\mathrm{H}-\mathrm{O}_{1}-\mathrm{H}\right)=13.6$ \\
\hline & & $\mathrm{O}_{1}-\mathrm{O}_{2}=2.64$ & $\mathrm{~S}^{\mathrm{Il}}-\left(\mathrm{H}-\mathrm{O}_{3}-\mathrm{H}\right)=8.6$ \\
\hline \multirow{8}{*}{$\begin{array}{l}\mathrm{OH} \text { ( } \mathrm{H} \text { up) }-\mathrm{HOH} \text { (H up) } \\
-\mathrm{H}_{2} \mathrm{O}-\mathrm{H} \text { (top) } \\
1 / 2 \mathrm{ML} \\
\text { Figure } 2 \mathrm{~h}\end{array}$} & 0.484 & $\mathrm{Pt}-\mathrm{O}_{1}=2.00$ & $\mathrm{H}-\mathrm{O}_{2}-\mathrm{H}=101.3$ \\
\hline & & & \\
\hline & & $\mathrm{Pt}-\mathrm{O}_{2}=2.91$ & $\mathrm{H}-\mathrm{O}_{3}-\mathrm{H}=104.1$ \\
\hline & & $\mathrm{Pt}-\mathrm{O}_{3}=2.51$ & $\mathrm{~S}^{\perp}-\left(\mathrm{O}_{2}-\mathrm{H}_{\mathrm{up}}\right)=5.2$ \\
\hline & & $\mathrm{Pt}-\mathrm{H}_{1}=1.55$ & $\mathrm{~S}^{\perp-}\left(\mathrm{Pt}-\mathrm{O}_{2}\right)=0.3$ \\
\hline & & $\mathrm{O}_{2 / 3}-\mathrm{H}=0.98,1.00$ & $\mathrm{~S}^{\perp}-\left(\mathrm{Pt}-\mathrm{O}_{1}\right)=2.9$ \\
\hline & & $\mathrm{O}_{1}-\mathrm{H}=0.99$ & $\mathrm{~S}^{\mathrm{Il}}-\left(\mathrm{O}_{1}-\mathrm{H}\right)=15.4$ \\
\hline & & $\mathrm{O}_{1}-\mathrm{O}_{2}=2.88$ & $\mathrm{~S}^{\mathrm{Il}}-\left(\mathrm{H}-\mathrm{O}_{3}-\mathrm{H}\right)=13.6$ \\
\hline \multirow{8}{*}{$\begin{array}{l}\mathrm{OH}(\mathrm{H} \mathrm{up})-\mathrm{HOH} \text { (H down) } \\
-\mathrm{H}_{2} \mathrm{O}-\mathrm{H} \text { (top) } \\
1 / 2 \mathrm{ML} \\
\text { Figure } 2 \mathrm{i}\end{array}$} & 0.518 & $\mathrm{Pt}-\mathrm{O}_{1}=1.99$ & $\mathrm{H}-\mathrm{O}_{2}-\mathrm{H}=100.0$ \\
\hline & & & \\
\hline & & $\mathrm{Pt}-\mathrm{O}_{2}=3.07$ & $\mathrm{H}-\mathrm{O}_{3}-\mathrm{H}=103.7$ \\
\hline & & $\mathrm{Pt}-\mathrm{O}_{3}=2.47$ & $\mathrm{~S}^{\mathrm{II}}-\left(\mathrm{O}_{2}-\mathrm{H}_{\mathrm{up}}\right)=15.7$ \\
\hline & & $\mathrm{Pt}-\mathrm{H}_{1}=1.54$ & $\mathrm{~S}^{\perp-}\left(\mathrm{Pt}-\mathrm{O}_{2}\right)=0.1$ \\
\hline & & $\mathrm{O}_{1 / 3}-\mathrm{H}=0.97-1.00$ & $\mathrm{~S}^{\perp-}\left(\mathrm{Pt}-\mathrm{O}_{1}\right)=3.1$ \\
\hline & & $\mathrm{O}_{2}-\mathrm{H}_{\text {up/down }}=0.98,1.00$ & $\mathrm{~S}^{\|}-\left(\mathrm{O}_{1}-\mathrm{H}\right)=18.2$ \\
\hline & & $\mathrm{O}_{1}-\mathrm{O}_{2}=2.91$ & $\mathrm{~S}^{\mathrm{II}}-\left(\mathrm{H}-\mathrm{O}_{3}-\mathrm{H}\right)=5.6$ \\
\hline
\end{tabular}

${ }^{a}$ In all cases the top Pt layer is relaxed. The binding energy $\left(E_{\text {bind }}\right)$ is with respect to the separated systems $\left[E(\mathrm{Pt}-\mathrm{slab}\right.$, relaxed $)+N \times E\left(\mathrm{H}_{2} \mathrm{O}\right), N=$ 1,2,3]. $\mathrm{H}_{2} \mathrm{O}$ denotes parallel water and $\mathrm{HOH}$ denotes water with $\mathrm{H}$ facing up or down (toward $\mathrm{Pt}$ ). Multiple indices denote equivalent atoms (e.g., $\mathrm{O}_{1 / 3}-\mathrm{H}$ $=0.98,1.00 \AA$ combines $\mathrm{O}_{1}-\mathrm{H}=0.98 \AA$ and $\mathrm{O}_{3}-\mathrm{H}=1.00 \AA$ ).

To check for a possible partial dissociation at a coverage of $1 / 2 \mathrm{ML}$ we first moved one $\mathrm{H}$ atom from $\mathrm{O}_{2}$-water $(\mathrm{HOH}$-water) to a position on top of an empty $\mathrm{Pt}$ atom, resulting in the $\mathrm{Pt} /$ $\mathrm{H}_{2} \mathrm{O}-\mathrm{OH}$ ( $\mathrm{H}$ up) $-\mathrm{H}_{2} \mathrm{O}-\mathrm{H}$ (on top) configuration (Figure $2 \mathrm{~g}$ ) with two waters, one hydroxyl, and a single hydrogen per surface unit cell. The energy of this partially dissociated structure is lower than the undissociated H-down structure by 0.20 and $0.06 \mathrm{eV}$ compared to the undissociated H-up case. Since the
$\mathrm{O}_{2}$-water that remains has only one hydrogen, it forms a covalent bond to the surface of $2.11 \AA$. This causes the $\mathrm{O}_{2}$-water to tilt out of the surface normal $\left(\mathrm{S}^{\perp}\right)$ by $5.5^{\circ}$ and increase its $\mathrm{H}-\mathrm{O}_{1}-\mathrm{H}$ angle to $112.4^{\circ}$ (compared to $107.5^{\circ}$ for $\mathrm{O}_{3}$-water). To form the hydrogen bond to the oxygen of the $\mathrm{OH}$ the $\mathrm{H}-\mathrm{O}_{1}-\mathrm{H}$ plane is tilted out of the surface plane by $13.6^{\circ}$. This finally leads to a flat structure within the $\mathrm{O}_{1}-\mathrm{O}_{2}-\mathrm{O}_{1}-\mathrm{O}_{2}$-chain [vertical oxygen displacement of $\Delta z\left(\mathrm{O}_{1}-\mathrm{O}_{2}\right)=0.03 \AA$ ] and a bilayer between 
Table 3. Binding Energies and Structural Information for Various Systems with $2 / 3 \mathrm{ML}$ Coverage ${ }^{a}$

\begin{tabular}{|c|c|c|c|c|}
\hline system (always $2 / 3 \mathrm{ML}$ on Pt) & top-Pt & $E_{\text {bind }}[\mathrm{eV}]$ & distances $[\AA ̊]$ & angles [deg] \\
\hline $\begin{array}{l}\mathrm{H}_{2} \mathrm{O}-\mathrm{HOH}(\mathrm{H} \text { up })-\mathrm{H}_{2} \mathrm{O}-\mathrm{HOH}(\mathrm{H} \text { up }) \\
\quad \text { Figure } 3 \mathrm{a}\end{array}$ & $\begin{array}{l}\text { fixed } \\
\text { relaxed }\end{array}$ & $\begin{array}{l}2.064 \\
2.021\end{array}$ & $\begin{array}{l}\mathrm{Pt}-\mathrm{O}_{2 / 4}=2.92,3.00 \\
\mathrm{Pt}-\mathrm{O}_{1 / 3}=2.46,2.50 \\
\mathrm{O}_{1 / 3}-\mathrm{H}=0.99 \\
\mathrm{O}_{2 / 4}-\mathrm{H}_{\text {up }}=0.97 \\
\mathrm{O}_{2 / 4}-\mathrm{H}_{\text {down }}=1.00 \\
\mathrm{O}-\mathrm{O}=2.77,2.86 \\
\boldsymbol{\Delta} \boldsymbol{z}\left(\mathbf{O}_{1 / 3}-\mathbf{O}_{2 / 4}\right)=\mathbf{0 . 4 2}\end{array}$ & $\begin{array}{l}\mathrm{H}-\mathrm{O}_{2 / 4}-\mathrm{H}=101.2,103.2 \\
\mathrm{H}-\mathrm{O}_{1 / 3}-\mathrm{H}=108.2,109.5 \\
\mathrm{~S}^{\perp}-\left(\mathrm{O}_{2 / 4}-\mathrm{H}_{\text {up }}\right)=6.5,8.7 \\
\mathrm{O}_{1 / 3}-\mathrm{O}_{4 / 2}-\mathrm{H}_{\text {down }}=10.6 \\
\mathrm{~S}^{\perp}-\left(\mathrm{Pt}-\mathrm{O}_{2 / 4}\right)=0.7 \\
\mathrm{~S}^{\perp}-\left(\mathrm{Pt}-\mathrm{O}_{1 / 3}\right)=1.0,3.4 \\
\mathrm{~S}^{\| l}-\left(\mathrm{H}-\mathrm{O}_{1 / 3}-\mathrm{H}\right)=-1.0,0.1\end{array}$ \\
\hline $\begin{array}{l}\mathrm{H}_{2} \mathrm{O}-\mathrm{HOH}(\mathrm{H} \text { down })-\mathrm{H}_{2} \mathrm{O}-\mathrm{HOH}(\mathrm{H} \text { down }) \\
\quad \text { Figure } 3 \mathrm{~b} \text { (most stable) }\end{array}$ & $\begin{array}{l}\text { fixed } \\
\text { relaxed }\end{array}$ & $\begin{array}{l}2.300 \\
2.389\end{array}$ & $\begin{array}{l}\mathrm{Pt}-\mathrm{O}_{2 / 4}=3.11 \\
\mathrm{Pt}-\mathrm{O}_{1 / 3}=2.50,2.54 \\
\mathrm{O}_{1 / 3}-\mathrm{H}=0.99 \\
\mathrm{O}_{2 / 4}-\mathrm{H}_{\text {up }}=0.99 \\
\mathrm{O}_{2 / 4}-\mathrm{H}_{\text {down }}=1.01 \\
\mathrm{O}-\mathrm{O}=2.79,2.82 \\
\boldsymbol{\Delta z}\left(\mathbf{O}_{1 / 3}-\mathbf{O}_{2 / 4}\right)=\mathbf{0 . 4 2}\end{array}$ & $\begin{array}{l}\mathrm{H}-\mathrm{O}_{2 / 4}-\mathrm{H}=99.5,99.8 \\
\mathrm{H}-\mathrm{O}_{1 / 3}-\mathrm{H}=105.7,105.8 \\
\mathrm{~S}^{\perp}-\left(\mathrm{O}_{2 / 4}-\mathrm{H}_{\text {down }}\right)=1.5,2.6 \\
\mathrm{O}_{1 / 3}-\mathrm{O}_{4 / 2}-\mathrm{H}_{\text {up }}=17.2 \\
\mathrm{~S}^{\perp}-\left(\mathrm{Pt}-\mathrm{O}_{2 / 4}\right)=0.6 \\
\mathrm{~S}^{\perp}-\left(\mathrm{Pt}-\mathrm{O}_{1 / 3}\right)=0.2,0.4 \\
\mathrm{~S}^{\| \prime}-\left(\mathrm{H}-\mathrm{O}_{1 / 3}-\mathrm{H}\right)=28.2\end{array}$ \\
\hline $\begin{array}{l}\mathrm{H}_{2} \mathrm{O}-\mathrm{OH}(\mathrm{H} \text { up })-\mathrm{H} \text { (top) }-\mathrm{H}_{2} \mathrm{O}-\mathrm{OH} \\
\quad(\mathrm{H} \text { up })-\mathrm{H} \text { (top) }\end{array}$ & fixed & 2.046 & & \\
\hline Figure 3c (most stable dissociated case) & relaxed & 2.127 & $\mathrm{Pt}-\mathrm{O}_{2 / 4}=2.11$ & $\mathrm{Pt}-\mathrm{O}_{2 / 4}-\mathrm{H}=100.1,101.4$ \\
\hline & & & $\begin{array}{l}\mathrm{Pt}-\mathrm{O}_{1 / 3}=2.20 \\
\mathrm{O}_{1 / 3}-\mathrm{H}=1.02 \\
\mathrm{O}_{2 / 4}-\mathrm{H}_{\text {up }}=0.98 \\
\mathrm{Pt}-\mathrm{H}_{\text {top }}=1.54 \\
\mathrm{O}_{1}-\mathrm{O}_{2}=2.62 \\
\mathrm{O}_{1}-\mathrm{O}_{4}=3.00 \\
\boldsymbol{\Delta z}\left(\mathbf{O}_{1 / 3}-\mathbf{O}_{2 / 4}\right)=\mathbf{0 . 0 8}\end{array}$ & $\begin{array}{l}\mathrm{H}-\mathrm{O}_{1 / 3}-\mathrm{H}=110.4,112.7 \\
\mathrm{O}_{1 / 3}-\mathrm{O}_{4 / 2}-\mathrm{H}_{\text {up }}=15.4 \\
\mathrm{~S}^{\perp}-\left(\mathrm{Pt}-\mathrm{O}_{2 / 4}\right)=2.7,5.2 \\
\mathrm{~S}^{\perp}-\left(\mathrm{Pt}-\mathrm{O}_{1 / 3}\right)=0.2,2.7 \\
\mathrm{~S}^{\perp}-\left(\mathrm{Pt}-\mathrm{H}_{\text {top }}\right)=0.7,2.2 \\
\mathrm{~S}^{\| \prime}-\left(\mathrm{H}-\mathrm{O}_{1 / 3}-\mathrm{H}\right)=13.2\end{array}$ \\
\hline $\begin{array}{l}\mathrm{H}_{2} \mathrm{O}-\mathrm{OH}(\mathrm{H} \text { down })-\mathrm{H} \text { (top) }-\mathrm{H}_{2} \mathrm{O}-\mathrm{OH} \\
\quad(\mathrm{H} \text { down })-\mathrm{H} \text { (top) }\end{array}$ & fixed & $\begin{array}{l}\text { both } \mathrm{H} \text { down } \\
\text { relax to } \mathrm{H} \text { up }\end{array}$ & & \\
\hline $\begin{array}{l}\mathrm{H}_{2} \mathrm{O}-\mathrm{OH}(\mathrm{H} \text { up })-\mathrm{H}(\text { sub } 1)-\mathrm{H}_{2} \mathrm{O}-\mathrm{OH} \\
(\mathrm{H} \text { up })-\mathrm{H} \text { (sub 1) }\end{array}$ & $\begin{array}{l}\text { relaxed } \\
\text { fixed }\end{array}$ & -1.273 & & \\
\hline Figure $3 d$ & relaxed & -0.078 & $\begin{array}{l}\mathrm{Pt}-\mathrm{O}_{2 / 4}=2.11 \\
\mathrm{Pt}-\mathrm{O}_{1 / 3}=2.24 \\
\mathrm{O}_{1 / 3}-\mathrm{H}=1.02 \\
\mathrm{O}_{2 / 4}-\mathrm{H}_{\text {up }}=0.98 \\
\mathrm{Pt}-\mathrm{H}_{\text {sub }}=1.76-1.84 \\
\mathrm{O}_{1}-\mathrm{O}_{2}=2.69,2.70 \\
\mathrm{O}_{1}-\mathrm{O}_{4}=2.97 \\
\boldsymbol{\Delta z}\left(\mathbf{O}_{1 / 3}-\mathbf{O}_{2 / 4}\right)=\mathbf{0 . 0 8}\end{array}$ & $\begin{array}{l}\mathrm{Pt}-\mathrm{O}_{2 / 4}-\mathrm{H}=103.2 \\
\mathrm{H}-\mathrm{O}_{1 / 3}-\mathrm{H}=111.6-111.7 \\
\mathrm{O}_{1 / 3}-\mathrm{O}_{4 / 2}-\mathrm{H}_{\text {up }}=10.1 \\
\mathrm{~S}^{\perp}-\left(\mathrm{Pt}-\mathrm{O}_{2 / 4}\right)=1.5 \\
\mathrm{~S}^{\perp}-\left(\mathrm{Pt}-\mathrm{O}_{1 / 3}\right)=2.7 \\
\mathrm{~S}^{\| l}-\left(\mathrm{H}-\mathrm{O}_{1 / 3}-\mathrm{H}\right)=14.3\end{array}$ \\
\hline $\begin{array}{l}\mathrm{H}_{2} \mathrm{O}-\mathrm{OH}(\mathrm{H} \text { down })-\mathrm{H}(\text { sub } 1)-\mathrm{H}_{2} \mathrm{O}-\mathrm{OH} \\
\quad(\mathrm{H} \text { down })-\mathrm{H}(\text { sub } 1)\end{array}$ & fixed & $\begin{array}{l}\text { both } \mathrm{H} \text { down } \\
\text { relax to } \mathrm{H} \text { up }\end{array}$ & & \\
\hline $\begin{array}{l}\mathrm{H}_{2} \mathrm{O}-\mathrm{OH}(\mathrm{H} \text { up })-\mathrm{H}(\text { sub } 2)-\mathrm{H}_{2} \mathrm{O}-\mathrm{OH} \\
(\mathrm{H} \text { up })-\mathrm{H}(\text { sub } 2)\end{array}$ & $\begin{array}{l}\text { relaxed } \\
\text { fixed }\end{array}$ & -0.627 & & \\
\hline Figure $3 \mathrm{e}$ & relaxed & 0.126 & $\begin{array}{l}\mathrm{Pt}-\mathrm{O}_{2 / 4}=2.11 \\
\mathrm{Pt}-\mathrm{O}_{1 / 3}=2.24,2.26 \\
\mathrm{O}_{1 / 3}-\mathrm{H}=1.02 \\
\mathrm{O}_{2 / 4}-\mathrm{H}_{\text {up }}=0.98 \\
\mathrm{Pt}-\mathrm{H}_{\text {sub }}=1.65-1.67 \\
\mathrm{O}_{1}-\mathrm{O}_{2}=2.67,2.70 \\
\mathrm{O}_{1}-\mathrm{O}_{4}=2.94 \\
\boldsymbol{\Delta z}\left(\mathbf{O}_{1 / 3}-\mathbf{O}_{2 / 4}\right)=\mathbf{0 . 7}-\mathbf{0 . 1 1}\end{array}$ & $\begin{array}{l}\mathrm{Pt}-\mathrm{O}_{2 / 4}-\mathrm{H}=102.0,102.2 \\
\mathrm{H}-\mathrm{O}_{1 / 3}-\mathrm{H}=111.4,111.5 \\
\mathrm{O}_{1 / 3}-\mathrm{O}_{4 / 2}-\mathrm{H}_{\mathrm{up}}=12.0 \\
\mathrm{~S}^{\perp}-\left(\mathrm{Pt}-\mathrm{O}_{2 / 4}\right)=1.3,2.1 \\
\mathrm{~S}^{\perp}-\left(\mathrm{Pt}-\mathrm{O}_{1 / 3}\right)=2.4,3.7 \\
\mathrm{~S}^{\| l}-\left(\mathrm{H}-\mathrm{O}_{1 / 3}-\mathrm{H}\right)=14.6\end{array}$ \\
\hline $\begin{array}{l}\mathrm{H}_{2} \mathrm{O}-\mathrm{OH}(\mathrm{H} \text { down })-\mathrm{H}(\text { sub } 2)-\mathrm{H}_{2} \mathrm{O}-\mathrm{OH} \\
\quad(\mathrm{H} \text { down })-\mathrm{H}(\text { sub } 2)\end{array}$ & fixed & $\begin{array}{l}\text { both } \mathrm{H} \text { down } \\
\quad \text { relax to } \mathrm{H} \text { up }\end{array}$ & & \\
\hline $\begin{array}{l}\mathrm{OH}(\mathrm{H} \text { up })-\mathrm{H}_{2} \mathrm{O}-\mathrm{H}(\text { sub } 3)-\mathrm{H}_{2} \mathrm{O}-\mathrm{OH} \\
\quad(\mathrm{H} \text { up })-\mathrm{H}(\text { sub } 3)\end{array}$ & $\begin{array}{l}\text { relaxed } \\
\text { fixed }\end{array}$ & -1.352 & & \\
\hline Figure $3 \mathrm{f}$ & relaxed & 0.089 & $\begin{array}{l}\mathrm{Pt}-\mathrm{O}_{1 / 4}=2.08,2.10 \\
\mathrm{Pt}-\mathrm{O}_{2 / 3}=2.26,2.32 \\
\mathrm{O}_{2 / 3}-\mathrm{H}=0.98-1.02 \\
\mathrm{O}_{1 / 4}-\mathrm{H}_{\text {up }}=0.98,1.00 \\
\mathrm{Pt}-\mathrm{H}_{\text {sub }}=1.61-1.73 \\
\mathrm{O}_{1}-\mathrm{O}_{2}=2.99 \\
\mathrm{O}_{1}-\mathrm{O}_{4}=2.81 \\
\boldsymbol{\Delta z}\left(\mathbf{O}_{1 / 3}-\mathbf{O}_{2 / 4}\right)=\mathbf{0 . 0 8}\end{array}$ & $\begin{array}{l}\mathrm{Pt}-\mathrm{O}_{1 / 4}-\mathrm{H}=99.5,104.6 \\
\mathrm{H}-\mathrm{O}_{2 / 3}-\mathrm{H}=109.6-110.6 \\
\mathrm{O}_{2}-\mathrm{O}_{1}-\mathrm{H}_{\mathrm{up}}=6.0 \\
\mathrm{O}_{1}-\mathrm{O}_{4}-\mathrm{H}_{\mathrm{up}}=13.4 \\
\mathrm{~S}^{\perp}-\left(\mathrm{Pt}-\mathrm{O}_{1 / 4}\right)=0.3 \\
\mathrm{~S}^{\perp}-\left(\mathrm{Pt}-\mathrm{O}_{2 / 3}\right)=3.3,3.4 \\
\mathrm{~S}^{\prime \prime}-\left(\mathrm{H}-\mathrm{O}_{1 / 3}-\mathrm{H}\right)=21.0\end{array}$ \\
\hline \multirow{2}{*}{$\begin{array}{l}\mathrm{H}_{2} \mathrm{O}-\mathrm{OH}(\mathrm{H} \text { up })-\mathrm{H}(\text { sub 3) } \\
-\mathrm{H}_{2} \mathrm{O}-\mathrm{OH}(\mathrm{H} \mathrm{up})-\mathrm{H}(\text { sub 3) } \\
\text { Figure } 3 \mathrm{~g}\end{array}$} & fixed & -1.392 & $\mathrm{Pt}-\mathrm{O}_{2 / 4}=2.12$ & $\mathrm{Pt}-\mathrm{O}_{2 / 4}-\mathrm{H}=102.0,102.1$ \\
\hline & relaxed & unstable & $\begin{array}{l}\mathrm{Pt}-\mathrm{O}_{1 / 3}=2.21 \\
\mathrm{O}_{1 / 3}-\mathrm{H}=1.01-1.02 \\
\mathrm{O}_{2 / 4}-\mathrm{H}_{\text {up }}=0.98 \\
\mathrm{Pt}-\mathrm{H}_{\text {sub }}=1.69-1.71 \\
\mathrm{O}_{1}-\mathrm{O}_{2}=2.70,2.71 \\
\mathrm{O}_{1}-\mathrm{O}_{4}=2.96 \\
\boldsymbol{\Delta} z\left(\mathbf{O}_{1 / 3}-\mathbf{O}_{2 / 4}\right)=\mathbf{0 . 1 3}-\mathbf{0 . 4 7}\end{array}$ & $\begin{array}{l}\mathrm{H}-\mathrm{O}_{1 / 3}-\mathrm{H}=112.1,112.2 \\
\mathrm{O}_{1 / 3}-\mathrm{O}_{4 / 2}-\mathrm{H}_{\mathrm{up}}=12.7 \\
\mathrm{~S}^{\perp}-\left(\mathrm{Pt}-\mathrm{O}_{2 / 4}\right)=2.2,2.5 \\
\mathrm{~S}^{\perp}-\left(\mathrm{Pt}-\mathrm{O}_{1 / 3}\right)=2.7 \\
\mathrm{~S}^{\| \prime}-\left(\mathrm{H}-\mathrm{O}_{1 / 3}-\mathrm{H}\right)=18.2\end{array}$ \\
\hline
\end{tabular}

${ }^{a} E_{\text {bind }}$ is with respect to the separated systems ( $E\left(\right.$ Pt-slab) $+4 \times E\left(\mathrm{H}_{2} \mathrm{O}\right)$ ), means per $4 \mathrm{H}_{2} \mathrm{O}$. In cases where only the water adsorbates were allowed to relax $E$ (Pt-slab) is the energy of the completely fixed slab. Otherwise $E\left(\mathrm{Pt}\right.$-slab) is for the slab with relaxed surface layer. $\mathrm{H}_{2} \mathrm{O}$ denotes water with both $\mathrm{H}$ oriented parallel to the surface, $\mathrm{HOH}$ with $\mathrm{H}$ facing up or down. Multiple indices denote equivalent atoms (e.g., $\mathrm{O}_{1 / 3}-\mathrm{H}=0.98,1.00 \AA$ combines $\mathrm{O}_{1}-\mathrm{H}$ $=0.98 \AA$ and $\mathrm{O}_{3}-\mathrm{H}=1.00 \AA$ ). 
$\mathrm{O}_{1 / 2^{-}}$and $\mathrm{O}_{3}$-water $\left(\Delta z\left(\mathrm{O}_{1 / 2}-\mathrm{O}_{3}\right)=0.17,0.20 \AA\right.$. We also dissociated one $\mathrm{H}$ atom from the parallel oriented $\mathrm{O}_{1}$-water, allowing the $\mathrm{O}_{2}$-water to have a $\mathrm{H}$-up or $\mathrm{H}$-down orientations. (The energy difference between the $\mathrm{H}$ up and the $\mathrm{H}$ down configuration is only $0.03 \mathrm{eV}$ ). Both of these partially dissociated structures are less stable than the corresponding undissociated by $0.94-1.06 \mathrm{eV}$, indicating that these structures would not be observed experimentally. The waters have structures similar to their undissociated analogues. Again, the $\mathrm{O}_{2}$-water has the $\mathrm{H}-\mathrm{O}-\mathrm{H}$ plane tilted from the surface parallel by $25.3^{\circ}$. This leads to a bilayer structure with the $\mathrm{O}$ atoms separated by 0.38 and $0.40 \AA$ for the $\mathrm{H}$ up structure and by 0.49 and $0.41 \AA$ for the $\mathrm{H}$ down configuration.

Finally, we added the fourth water to the SUC, leading to the $2 / 3$ ML coverage established experimentally as the saturated single overlayer. ${ }^{9,10}$ Systems with this amount of water are shown in Figure 3, whereas the energetics and structures are in Table 3.

The saturated water layer forms a hexagonal structure with half the water in the $\mathrm{H}_{2} \mathrm{O}$ configuration (oriented almost parallel to the surface) forming a direct donor-acceptor bond to the surface $\left(\mathrm{O}_{1 / 3}\right.$-water $)$, and the other half has the $\mathrm{HOH}$ configuration $\left(\mathrm{H}-\mathrm{O}-\mathrm{H}\right.$ plane perpendicular to the surface, $\mathrm{O}_{2 / 4}$-water). These two types of water alternate to form a layer in which each $\mathrm{H}_{2} \mathrm{O}$ makes 3 hydrogen bonds. We find the most stable structure to have the $\mathrm{O}_{2}$ - and $\mathrm{O}_{4}$-water oriented so that the hydrogen makes an agostic bond to a Pt atom (H-down). This is $0.37 \mathrm{eV}$ per SUC more stable than the H-up orientation. The $\mathrm{H}$-down structure leads to $\mathrm{O}_{2}$ - and $\mathrm{O}_{4}$-water with $\mathrm{H}-\mathrm{O}-\mathrm{H}$ angles of $99.7^{\circ}$, significantly less than the gas-phase molecule $\left(104.7^{\circ}\right)$, whereas the $\mathrm{O}_{1}$ - and $\mathrm{O}_{3}$-water open up to $105.8^{\circ}$. The orientation of the $\mathrm{H}-\mathrm{O}-\mathrm{H}$ planes in $\mathrm{O}_{2 / 4}$-water is tilted $28.2^{\circ}$ away from the surface (as observed for lower coverage). The vertical distance $\mathrm{O}_{1 / 3}-\mathrm{O}_{2 / 4}$ is $0.42 \AA$ for either orientation of the $\mathrm{H}$ atoms. This is in contrast to the calculations by Meng et al., ${ }^{11}$ who found a difference between the vertical $\mathrm{O}-\mathrm{O}$ distances for $\mathrm{H}$ up $[\Delta z(\mathrm{O}-\mathrm{O})=0.63 \AA]$ and $\mathrm{H}$ down $[\Delta z(\mathrm{O}-\mathrm{O})=0.35]$ by $0.28 \AA$. The $\mathrm{O}-\mathrm{O}$ distances in the $\mathrm{HB}$ are $2.82 \AA$ for $\mathrm{H}$-up and $2.70 \AA$ for $\mathrm{H}$-down. In addition, the $\mathrm{H}_{2} \mathrm{O}$ molecules (parallel to surface) of the $\mathrm{H}$-down structure have $\mathrm{Pt}-\mathrm{O}$ distances of 2.50 and $2.54 \AA$, whereas the agostic $\mathrm{Pt}-\mathrm{HO}$ distances are both $3.11 \AA$ A. Meng et al. ${ }^{11}$ report distances similar to ours ( 2.68 for parallel $\mathrm{H}_{2} \mathrm{O}$ and $3.14 \AA$ for $\mathrm{HOH}$ ). Although, they could not distinguish between $\mathrm{H}$-up and $\mathrm{H}$-down their $\mathrm{H}$-down calculations show good agreement with vibrational frequencies from HREELS studies. Our general agreement with Meng et al. despite the differences in the method (exchange-correlation functional and basis-set representation) suggests reliable structures. However, our results for parallel water lead to $\approx 0.2 \AA$ shorter $\mathrm{Pt}-\mathrm{O}$ distances. Since Ogasawara et al. in their calculations achieved general agreement with the measured XAS and XPS spectra only after shortening the $\mathrm{Pt}-\mathrm{O}$ distance to $2.3-2.4 \AA$ (see Introduction), our calculations ( $\mathrm{Pt}-\mathrm{O}_{1 / 3}=2.50,2.54 \AA$ ) might lead to a better description of the XAS and XPS spectra.

Although the partially dissociated case has already been contradicted by Ogasawara et al., we calculated the partially dissociated structure to evaluate energetical and geometrical differences to the most stable $\mathrm{H}$-down system. Each $\mathrm{O}_{2 / 4}$-water molecule dissociates one $\mathrm{H}$ atom to form a $\mathrm{Pt}-\mathrm{OH}$ covalent bond. The dissociated hydrogen atoms bind on top of the remaining free $\mathrm{Pt}$ atoms, so that every surface $\mathrm{Pt}$ is bonded either to $\mathrm{H}$, to $\mathrm{OH}$, to the $\mathrm{O}$ of $\mathrm{H}_{2} \mathrm{O}$ or to the $\mathrm{HO}$ of $\mathrm{HOH}$. We optimized structures starting with the $\mathrm{H}$ up or the $\mathrm{H}$ down atoms dissociated. Moving the $\mathrm{H}$ up atoms to the empty Pt site reoriented the hydroxyl molecules such that each $\mathrm{OH}$ forms a covalent $\mathrm{Pt}-\mathrm{OH}$ bond $(2.20 \AA)$. The single $\mathrm{H}$ atom remains on top of the Pt atom (1.54 $\AA$ ), which is the most stable position calculated using a 35 atom three-layer Pt cluster (zero coverage limit and neglecting zero point energy, which changes the preference to a $\mu_{3}$ site). ${ }^{25}$ The adsorption energy (with respect to the plain slab and 4 separated waters) per surface unit cell is $2.13 \mathrm{eV}$, which is $0.11 \mathrm{eV}$ more stable than the undissociated structure with $\mathrm{H}$ facing up, but $0.26 \mathrm{eV}$ less stable than the undissociated structure with $\mathrm{H}$ facing down. This confirms the conclusion by Ogasawara et al. ${ }^{6}$ and Meng et al. ${ }^{11}$ that the partial dissociation of water reported for $\mathrm{Ru}(0001)$ is unfavorable for $\mathrm{Pt}(111)$. Moreover, the binding through alternating $\mathrm{Pt}-\mathrm{O}$ and $\mathrm{Pt}-\mathrm{HO}$ bonds agrees with the conclusions of Ogasawara et al. However, we do not find the nearly flat $\mathrm{O}$ plane (displacement $0.25 \AA$ ) for this structure proposed by Ogasawara. Instead, the $\mathrm{H}$ down structure leads to larger oxygen displacements of 0.42 $\AA$ (comparable to $0.35 \AA$ proposed by Meng et al.), essentially the same as we find for H-up. In contrast, we find that the partially dissociated case leads to oxygen displacements $<0.08$ $\AA$. This is a consequence of the covalent bond to the hydroxyl, which pulls the oxygens closer to the surface (from $\approx 3.11 \AA$ to $2.11 \AA$ ). Since the neighboring $\mathrm{O}_{1}$ - and $\mathrm{O}_{3}$-water are already closer to the surface (2.54 $\AA$ ) and tend to optimize the H-bonds, they are "towed" toward the surface $(2.20 \AA)$. The dissociated hydrogen donates a partial charge of $0.16 \mathrm{e}$ to the surface. ${ }^{26}$

With no water or $\mathrm{OH}$ on the surface, the energy barrier for $\mathrm{H}$ to hop between different surface binding sites on $\operatorname{Pt}(111)$ is $<0.1 \mathrm{eV},{ }^{25,27}$ making it quite mobile. We considered that there might be only a small barrier for hydrogen to migrate to a subsurface site, and hence, we examined the partially dissociated configuration in which both hydrogens are in subsurface sites between the first and second Pt surface layers. We examined three different structures: both $\mathrm{H}$ atoms in the tetrahedral interstitial site next to the hydroxyl (subsurface 1), both $\mathrm{H}$ in the octahedral interstitial site between the first and second layer (subsurface 2), and both $\mathrm{H}$ in the tetrahedral interstitial site beneath the Pt atoms to which the hydroxyls are bound (subsurface 3). Locating the $\mathrm{H}$ atoms in subsurface 1 positions leads to a negative binding energy of $-0.08 \mathrm{eV}$ (endothermic) and a structure of the water overlayer comparable to the dissociated configuration with $\mathrm{H}$ on surface. The subsurface $\mathrm{Pt}-\mathrm{H}$ bonds of the tetrahedral site are $1.76-1.84 \AA$, much longer than the surface $\mathrm{Pt}-\mathrm{H}$ bond of $1.54 \AA$. The result is that the Pt surface deforms only slightly by the presence of the subsurface hydrogens. Similar behavior was observed for the other subsurface systems.

We also started minimizations with the $\mathrm{H}$ at the octahedral positions beneath fcc-sites. However, both $\mathrm{H}$ atoms moved to positions directly beneath the empty surface Pt atoms (subsurface 2), leading to a net binding energy of $0.13 \mathrm{eV}$ per SUC. Positioning the $\mathrm{H}$ atoms at the subsurface 3 sites, leads to two possible structures of the water and hydroxyl molecules, while

(25) Jacob, T.; Goddard, W. A., III. J. Phys Chem. B 2003, submitted.

(26) Jacob, T.; Goddard, W. A., III., in preparation.

(27) Olsen, R. A.; Kroes, G. J.; Baerends, E. J. J. Chem. Phys. 1999, 111(24), 11155 . 
all subsurface hydrogens remain in the tetrahedral site. One structure $\left[\mathrm{Pt} / \mathrm{OH}\left(\mathrm{H}\right.\right.$ up) $-\mathrm{H}_{2} \mathrm{O}-\mathrm{H}$ (subsurface 3$)-\mathrm{H}_{2} \mathrm{O}-\mathrm{OH}(\mathrm{H}$ up)-H (subsurface 3)] has an energy comparable to that for subsurface 1 and subsurface 2 positions, with a binding energy of $0.08 \mathrm{eV}$ (first surface layer relaxed). The subsurface $\mathrm{H}$ disturbs the electronic configuration so that the $\mathrm{O}_{1}$-water gives one of its hydrogens to the $\mathrm{O}_{2}$-hydroxyl, leading to modified surface positions. Thus, not every water is next to a hydroxyl and vice versa $\left(\mathrm{H}_{2} \mathrm{O}-\mathrm{OH}-\mathrm{H}_{2} \mathrm{O}-\mathrm{OH}\right.$ ordering $)$, but is next to another water $\left(\mathrm{H}_{2} \mathrm{O}-\mathrm{OH}-\mathrm{OH}-\mathrm{H}_{2} \mathrm{O}\right.$ ordering, see Figure $\left.3 \mathrm{f}\right)$.

The studies presented here address the structure of water on $\mathrm{Pt}(111)$ at various coverages. We find that the layer saturates at $2 / 3 \mathrm{ML}$ with the half of the waters parallel to the surface $\left(\mathrm{H}_{2} \mathrm{O}\right)$ and bonding on top a $\mathrm{Pt}$ through an $\mathrm{O}$ lone pair, while the other half $(\mathrm{HOH})$ has one $\mathrm{H}$ pointing toward a $\mathrm{Pt}$ atom (an agostic bond) and the other $\mathrm{H}$ serving as a donor hydrogen bond to one neighboring $\mathrm{H}_{2} \mathrm{O}$-water. This structure is $0.37 \mathrm{eV}$ per SUC more stable than the $\mathrm{H}$ up structure. Both lead to a separation between the planes of oxygens of $0.42 \AA$. In addition, we find that the cost of dissociating the $\mathrm{HOH}$ half of the waters to form $\mathrm{Pt}-\mathrm{OH}$ and $\mathrm{Pt}-\mathrm{H}$ covalent bonds is only $0.26 \mathrm{eV}$ per SUC. This leads to a nearly flat structure with the $\mathrm{O}$ planes differing by $0.08 \AA$ in the vertical direction.

It is useful to relate the total energies calculated here to simple additive quantities. On the basis of the lower coverage structures, we assume that binding an isolated parallel $\mathrm{H}_{2} \mathrm{O}$ to surface through the lone pair is $\mathrm{Pt}-\mathrm{H}_{2} \mathrm{O}=0.38 \mathrm{eV}$. Then for the $\mathrm{H}$-up bilayer with a total binding energy of $2.02 \mathrm{eV}$ and 6 hydrogen bonds per SUC, we write

$$
2.02=2 \times 0.38+6 \mathrm{HB}
$$

leading to $\mathrm{HB}=0.21 \mathrm{eV}=4.8 \mathrm{kcal} / \mathrm{mol}$, very close to the bond energy of water dimer, $5.02 \mathrm{kcal} / \mathrm{mol}$. Then considering the more stable H-down bilayer, we get

$$
2.39=2 \times 0.76+6 \mathrm{HB}+2 \mathrm{Pt}-\mathrm{HOH}
$$

where the agostic bond is $\mathrm{Pt}-\mathrm{HOH}=0.20 \mathrm{eV}=4.6 \mathrm{kcal} / \mathrm{mol}$. These numbers are similar to the results for the $1 / 3 \mathrm{ML}$ with $\mathrm{HB}$ smaller by $0.07 \mathrm{eV}$ and $\mathrm{Pt}-\mathrm{HOH}$ stronger by $0.02 \mathrm{eV}$. Including these numbers in the estimates of the cluster energies on $\mathrm{Pt}$, suggests that the tetramer would be stable by $0.66 \mathrm{eV} /$ $\mathrm{H}_{2} \mathrm{O}$, whereas the 13 molecule cluster would be stable by 0.53 $\mathrm{eV} / \mathrm{H}_{2} \mathrm{O}$.

The result is a net binding energy per water of $0.60 \mathrm{eV}=$ $13.8 \mathrm{kcal} / \mathrm{mol}$, just above our predicted average energy for a 13 molecule isolated cluster. This is much more stable than bulk ice $(\sim 12.2 \mathrm{kcal} / \mathrm{mol}=0.53 \mathrm{eV})$ or bulk water $(\sim 10.8 \mathrm{kcal} / \mathrm{mol}$ $=0.47 \mathrm{eV}) .{ }^{28}$ In contrast, the $\mathrm{H}$-up bilayer structure leads to a net binding energy per water of $0.51 \mathrm{eV}=11.54 \mathrm{kcal} / \mathrm{mol}$, much more compatible with the energy of bulk water.

(28) Gray, D. E. American Institute of Physics Handbook; McGraw-Hill: New York, 1972.
Adding an isolated additional water to the full bilayer is expected to lead to much a much weaker interaction of $\sim 1 \mathrm{HB}$ $\approx 0.21 \mathrm{eV}=4.8 \mathrm{kcal} / \mathrm{mol}$, which is too weak to bind at $300 \mathrm{~K}$. This indicates that the first layer of water on Pt surface forms a stable film that may impede the reactions and may lead to particularly weak interactions with additional water. Probably, the LEED pattern for our structure would resemble that of the full-terminated water layers observed on thicker $(10 \AA)$ ice layers by Materer et al. on $\mathrm{Pt}(111) .{ }^{29}$ Perhaps HREELS or IR studies of the $\mathrm{OH}$ intensities and shifts would provide data to distinguish between $\mathrm{H}$-up and $\mathrm{H}$-down bilayers. Of course the presence of cations and anions can dramatically change the stability and structure of this first layer.

We should emphasize that we have considered only the enthalpies of binding; including entropy could lead to temperature-dependent effects important in determining the observed phases and kinetics.

\section{Summary}

We find that the lowest energy structure for a saturated layer of water on Pt is the H-down monolayer of Figure 1b. This is stabilized over the traditional $\mathrm{H}$-up bilayer by the agostic interactions $(\sim 0.20 \mathrm{eV}=4.6 \mathrm{kcal} / \mathrm{mol})$ of the $\mathrm{HOH}$ over the upper half the water to the empty Pt sites. The lower half of the water bilayer forms a donor-acceptor bond through the $\mathrm{O}$ lone pair, and within the bilayer each water forms hydrogen bonds (each $0.213 \mathrm{eV}=4.9 \mathrm{kcal} / \mathrm{mol}$ ) to three surrounding waters. The net is an average energy per water of $0.60 \mathrm{eV}=$ $13.8 \mathrm{kcal} / \mathrm{mol}$.

We find that for the monolayer, dissociating the upper half of the water (agostically bonded to the surface) to form an $\mathrm{OH}$ covalent bond to the surface and an $\mathrm{H}$ bonding to the $1 / 3$ of the Pt sites still empty in the bilayer structure leads to a net surface film that is weaker by only $0.066 \mathrm{eV} /$ water $=1.51 \mathrm{kcal} / \mathrm{mol}$ per water. Although not the most stable this is certainly close enough that such dissociated water may play important roles in the chemistry of this first layer of water on Pt. Moreover, these results should have implications in the catalytic properties of electrocatalysis on Pt.

Acknowledgment. We thank Prof. P. A. Thiel of Iowa State University for fruitful discussions and Dr. Peter Schultz from Sandia National Labs for the use of the SeqQuest PBC DFT code. T.J. gratefully acknowledges support by the German academic exchange service (DAAD). Additional support was provided by GM-GAPC and NSF-NIRT. The computation facilities of the MSC have been supported by grants from ARODURIP, ONR-DURIP, NSF (MRI, CHE), and IBM-SUR. In addition the MSC is supported by grants from DoE ASCI, AROMURI, ARO-DARPA, ONR-MURI, NIH, ONR, General Motors, ChevronTexaco, Seiko-Epson, Beckman Institute, and Asahi Kasei.

\section{JA049920Y}

(29) Materer, N.; Starke, U. Barbieri, A., Van Hove, M. A.; Somorjai, G. A Kroes, G. J.; Minot, C. Surf. Sci. 1997, 381(2-3), 190. 